\title{
How I diagnose and treat chronic myelomonocytic leukemia
}

Mrinal M. Patnaik

Division of Hematology, Department of Internal Medicine, Mayo Clinic, Rochester, MN, USA

\author{
Correspondence: \\ Mrinal M. Patnaik \\ patnaik.mrinal@mayo.edu \\ Received: December 20, 2021 \\ Accepted: $\quad$ February 24, 2022. \\ Prepublished: March 3, 2022. \\ https://doi.org/10.3324/haematol.2021.279500 \\ @2022 Ferrata Storti Foundation \\ Haematologica material is published under \\ a CC-BY-NC license @) @ $\Theta$
}

\begin{abstract}
Chronic myelomonocytic leukemia (CMML) is a myelodysplastic syndrome/myeloproliferative overlap neoplasm characterized by sustained peripheral blood monocytosis and an inherent risk for transformation to acute myeloid leukemia (15-30\% over 3-5 years). While CMML is morphologically classified into CMML-0, 1 and 2 based on peripheral blood and bone marrow promonocyte/blast counts, a more clinically relevant classification into dysplastic and proliferative subtypes, based on the presenting white blood cell count, is helpful in prognostication and therapeutics. CMML is a neoplasm associated with aging, occurring on the background of clonal hematopoiesis, with TET2 and SRSF2 mutations being early initiating events. The subsequent acquisitions of $A S X L 1, R U N X 1$, SF3B1 and DNMT3A mutations usually give rise to dysplastic CMML, while ASXL1, JAK2V617F and RAS pathway mutations give rise to proliferative CMML. Patients with proliferative $C M M L$ have a more aggressive course with higher rates of transformation to acute myeloid leukemia. Allogeneic stem cell transplant remains the only potential cure for CMML; however, given the advanced median age at presentation (73 years) and comorbidities, it is an option for only a few affected patients (10\%). While DNA methyltransferase inhibitors are approved for the management of CMML, the overall response rates are 40-50\%, with true complete remission rates of $<20 \%$. These agents seem to be particularly ineffective in proliferative CMML subtypes with RAS mutations, while the TET2mutant/ASXL1wildtype genotype seems to be the best predictor for responses. These agents epigenetically restore hematopoiesis in responding patients without altering mutational allele burdens and progression remains inevitable. Rationally derived personalized/targeted therapies with disease-modifying capabilities are much needed.
\end{abstract}

\section{Introduction}

Chronic myelomonocytic leukemia (CMML) is a myeloid neoplasm characterized by sustained peripheral blood monocytosis (absolute monocyte count $\geq 1 \times 10^{9} / \mathrm{L}$, with monocytes accounting for $\geq 10 \%$ of the white blood cells), predominantly arising in the context of age-related clonal hematopoiesis, with overlapping features of myelodysplastic syndromes (MDS) and myeloproliferative neoplasms (MPN). ${ }^{1-3}$ The exact incidence and prevalence rates for $C M M L$ are hard to define, with Surveillance, Epidemiology, and End Results (SEER) registry data demonstrating an incidence of 0.4 cases per 100,000, with most studies showing a clear male preponderance. ${ }^{4-7}$ The median age of presentation for CMML patients is between 70-75 years, with "young CMML" patients being operationally defined as those who present at $<65$ years of age. ${ }^{4-8}$.

At the genome level CMML is relatively homogeneous, demonstrating approximately 10-12 somatic variants per kilobase of coding region, with most pathogenic variants involving TET2 (60\%), ASXL1 (40\%), SRSF2 (50\%) and RAS pathway (30\%) genes. However, clinically the disease is very heterogenous in presentation and outcomes, making diagnostic, prognostic and therapeutic decision-making challenging. ${ }^{2,3,9-11}$ Broadly, CMML can be classified into dysplastic CMML (dCMML), presenting with cytopenias and clinical signs and symptoms related to the same (fatigue, bruising and transfusion dependence) and proliferative CMML ( $\mathrm{pCMML}$ ), presenting with significant myeloproliferation, extramedullary hematopoiesis and associated constitutional symptoms (fever, weight loss, night sweats, anorexia, pruritus, bone pain and cachexia).$^{10}$ From a classification perspective, for several years CMML was classified as a subtype of MDS, with the World Health Organization (WHO) rightfully and formally classifying CMML as an MDS/MPN overlap neoplasm, from 2002 on- 
wards. ${ }^{1}$ In 2015, the International Working Group for MDS/MPN overlap neoplasms proposed CMML-specific disease response criteria, providing support for the recognition of CMML as a specific disease entity and providing impetus for CMML-specific clinical trials. ${ }^{12}$ These changes have clearly incentivized the development of disease-specific diagnostic, prognostic, and therapeutic strategies for patients with CMML. In this review, I discuss my approach to the diagnosis, prognosis, and management of patients with CMML.

\section{Diagnosis and differential diagnosis of chronic myelomonocytic leukemia:}

CMML is a neoplasm associated with aging, often arising in the background of clonal hematopoiesis (bi-allelic TET2, or TET2/SRSF2 mutations), with the subsequent acquisition of mutations involving signaling (RAS pathway or
JAK2V617F), epigenetic regulation (SETBP1, DNMT3A and $E Z H 2)$, transcription factors ( $R U N X 1)$ and pre mRNA splicing (SF3B1 and U2AF1), shaping clinical phenotypes (Figure 1). ${ }^{2,3,13}$ There are $P C M M L$ subtypes in which oncogenic RAS pathway mutations (NRAS, CBL, KRAS and PTPN11) are clear initiating driver mutations, occurring early in the course of disease, associated with poor outcomes (decreased survival and higher rates of transformation to acute myeloid leukemia [AML]) (Figure 1). ${ }^{10,14}$ Unlike in MDS, MPN or AML, TP53 mutations are extremely infrequent in CMML $(<1 \%)$, and are only really encountered at the time of CMML to AML transformation, or in the context of therapy-related CMML. $11,15,16$

The 2016 iteration of the WHO classification of myeloid neoplasms has outlined diagnostic criteria for CMML which include the presence of sustained peripheral blood monocytosis, absence of reactive causes, the presence of $<20 \%$ blasts and promonocytes (blast equivalents) in the peripheral blood and bone marrow, and exclusion of molecularly

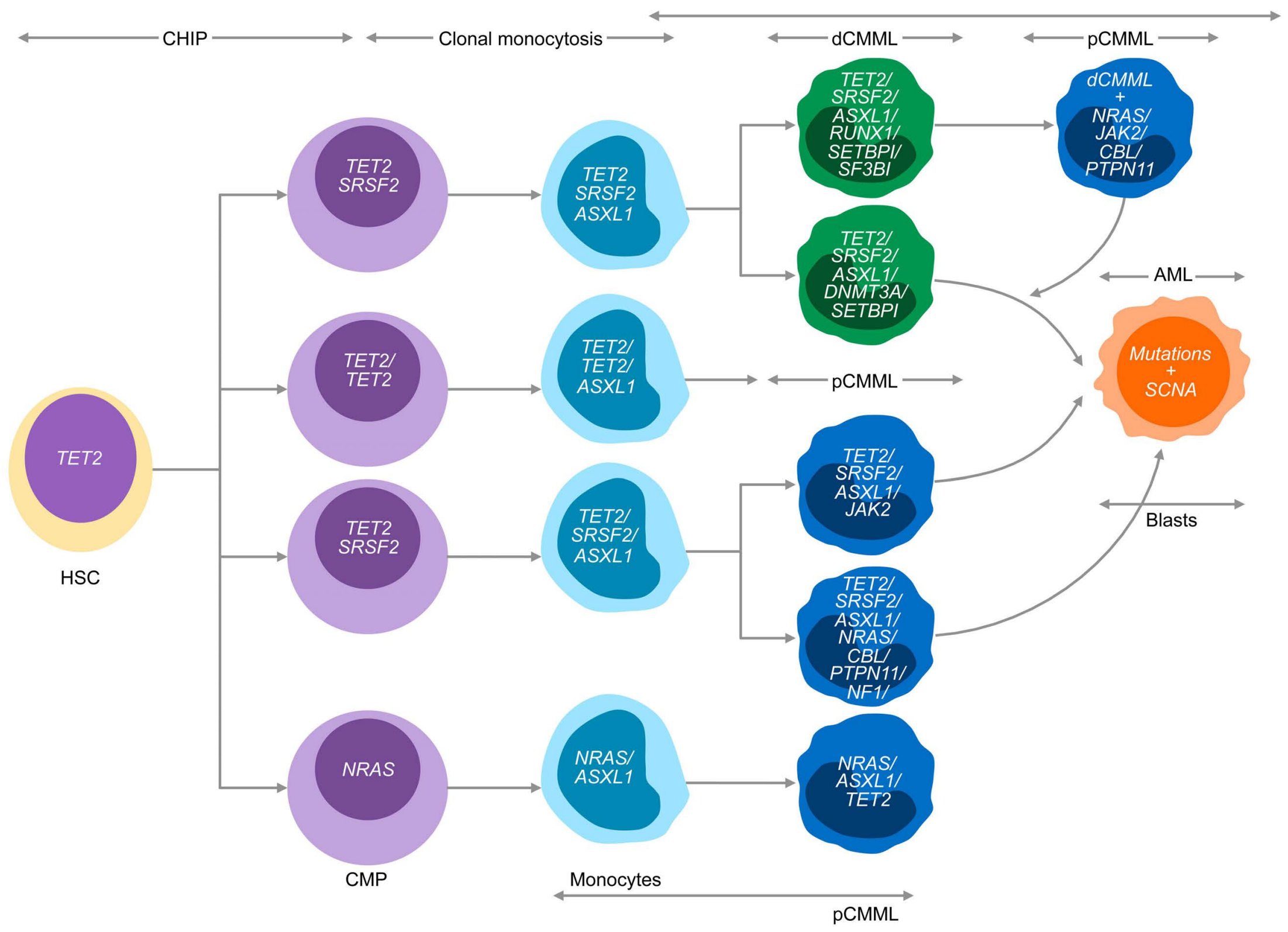

Figure 1. Clonal evolutionary dynamics in patients with chronic myelomonocytic leukemia. The dynamics of clonal evolution in patients with chronic myelomonocytic leukemia (CMML) demonstrating the early acquisition of TET2 and SRSF2 mutations in hematopoietic stem cells and common myeloid progenitor cells, followed by acquisition of signaling mutations (NRAS, KRAS, CBL, PTPN11, JAK2V617F), mutations in additional epigenetic regulator genes (ASXL1, EZH2, DNMT3A) and splicing components (SF3B1, U2AF1) resulting in dysplastic and proliferative subtypes of CMML. HSC: hematopoietic stem cell; CMP: common myeloid progenitor cell; CHIP: clonal hematopoiesis of indeterminate potential; dCMML: dysplastic CMML; $\mathrm{PCMML:proliferative} \mathrm{CMML;}$ AML: acute myeloid leukemia; SCNA: somatic copy number alterations. 
Table 1. 2017, World Health Organization criteria for the diagnosis of chronic myelomonocytic leukemia.

1 Persistent peripheral blood monocytosis $\geq 1 \times 10^{9} / \mathrm{L}$, with monocytes accounting for $\geq 10 \%$ of the white blood cell count

2 Not meeting criteria for $B C R:: A B L 1$ rearranged chronic myeloid leukemia, primary myelofibrosis, polycythemia vera and essential thrombocythemia *

3 No evidence for PDGFRA, PDGFRB, or FGFR1 rearrangements or the PCM1-JAK2 fusion

$4<20 \%$ blasts in the peripheral blood and bone marrow**

5 Dysplasia in one or more myeloid lineages. If myelodysplasia is absent or minimal, the diagnosis of CMML can still be made if the other requirements are met and

6 An acquired clonal cytogenetic or molecular genetic abnormality is present in hematopoietic cells ${ }^{\star \star *}$, or

7 The monocytosis has persisted for $>3$ months and all other causes of monocytosis have been excluded

*Cases of myeloproliferative neoplasms can be associated with monocytosis or monocytosis can develop during the disease. These cases may simulate chronic myelomonocytic leukemia (CMML). In these rare instances, a previous documented history of myeloproliferative neoplasm (MPN) excludes CMML, whereas the presence of MPN features in the bone marrow and/or of MPN-associated mutations (JAK2, CALR, or MPL) tend to support MPN with monocytosis rather than CMML. **Blasts and blast equivalents include myeloblasts, monoblasts, and promonocytes. Promonocytes are monocytic precursors with abundant light gray or slightly basophilic cytoplasm with a few scattered, fine lilaccolored granules, finely distributed, stippled nuclear chromatin, variably prominent nucleoli, and delicate nuclear folding or creasing. Abnormal monocytes, which can be present both in the peripheral blood and bone marrow, are excluded from the blast count. $* \star \star$ Presence of mutations in genes often associated with CMML (e.g., TET2, SRSF2, ASXL1, and SETBP1) in the proper clinical context can be used to support a diagnosis. It should be noted however, that many of these mutations can be age-related or be present in subclones. Therefore, caution would have to be used in the interpretation of these genetic results.

defined myeloid neoplasms that can present with monocytosis (BCR-ABL1, PDGFRA, PDGFRB, FGFR1 and PCM1-JAK2 rearrangements), with or without dysplasia (Table 1$).{ }^{1}$ In the absence of dysplasia, a diagnosis of CMML can be made if the monocytosis has persisted for $\geq 3$ months, reactive causes have been excluded, or somatic cytogenetic or molecular markers frequent in CMML (e.g., ASXL1, TET2, SRSF2 and SETBP1) can be documented. While this approach is very reasonable, there are limitations and important nuances associated with these criteria.

While absolute monocytosis is uncommon in chronic myeloid leukemia, it can occur in BCR-ABL1 p190 isoformdriven disease and, regardless of the presence or absence of a "myelocyte bulge", assessment for BCR-ABL1 fusions by fluorescence in situ hybridization, cytogenetics, and/or molecular techniques should be pursued. ${ }^{17}$ Chromosomal translocations/rearrangements involving PDGFRA and $P D G F R B$ can give rise to myeloid neoplasms, often characterized by prominent eosinophilia and responsiveness to imatinib. ${ }^{18-20}$ Among these, PDGFRB-rearranged myeloid neoplasms can be associated with absolute monocytosis ( $<1 \%$ of all cases morphologically diagnosed as CMML), usually with concomitant eosinophilia, and given their unique responsiveness to imatinib are best classified as molecularly defined neoplasms and not as CMML. ${ }^{18-20}$ More than 20 fusion partner genes have been described with $P D G F R B$, with $\mathrm{t}(5 ; 12)(\mathrm{q} 31-\mathrm{q} 32 ; \mathrm{p} 13)$, giving rise to the ETV6(TEL)-PDGFRB fusion, being the most common. ${ }^{21}$ The FIP1L1-PDGFRA fusion arising due to the CHIC2 deletion is the most common PDGFRA aberration and is uncommonly associated with monocytosis. ${ }^{19}$ While most $P D G F R B$ re- arrangements can be identified by conventional karyotyping, the FIP1L1-PDGFRA fusion is karyotypically occult and can only be detected by fluorescence in situ hybridization or molecular analyses. Similarly, FGFR1 and PCM1-JAK2 rearrangements are very uncommon causes of monocytosis and are more commonly associated with eosinophilia. Monocytosis can occur in the context of other myeloid neoplasms such as MDS and MPN and is associated with poor outcomes in MPN. ${ }^{22,23}$ While monocytosis in MDS can be a reflection of an ongoing evolutionary trajectory to CMML (oligo-monocytic CMML), in MPN, the utilization of monocyte repartitioning flow cytometry (discussed below) and driver mutation status can help differentiate CMML from MPN with monocytosis. ${ }^{23,24}$ Among classical MPNdriver mutations, while JAK2V617F can occur in $10 \%$ of CMML patients, mutations involving MPL and CALR are extremely infrequent and their detection should raise questions with regards to a bona fide $\mathrm{CMML}$ diagnosis. ${ }^{3,25}$ Occasionally NPM1 and FLT3 driver mutations are identified in CMML patients with excess blasts (5-19\%). ${ }^{26,27}$ For all practical purposes I consider these cases as acute myelomonocytic leukemia in evolution and treat them as such.

Reactive monocytosis is very common in practice and while viral infections and recovering bone marrow (from injury, drugs or chemotherapy) are frequent causes, sustained reactive monocytosis is more common in chronic infections such as subacute bacterial endocarditis, tuberculosis, brucellosis, leishmaniasis and leprosy and in autoimmune/inflammatory disorders such as systemic lupus erythematosus, sarcoidosis and mixed connective tissue 
disorder. ${ }^{28}$ Reactive monocytosis can also be seen in the context of metastatic visceral neoplasms, either due to enhanced mobilization of monocytes from the bone marrow, or due to increased monopoiesis mediated by CCL2 (C-C motif chemokine ligand 2). ${ }^{29}$

\section{Role of flow cytometry, next-gener- ation sequencing and bone marrow biopsies in the diagnosis of chronic myelomonocytic leukemia}

Conventional flow cytometry has a limited diagnostic role in CMML, given that immature/neoplastic monocytes do not express unique surface markers and that promonocytes/monoblasts are frequently CD34-negative (blast marker). ${ }^{30}$ Flow abnormalities that can be detected in CMML include abnormal myeloid maturation patterns involving CD11b, CD13 and CD16, along with aberrant expression of CD56 on monocytes. ${ }^{30}$ Monocyte repartitioning by flow cytometry has gained popularity, especially given its ability to differentiate CMML from other reactive and clonal causes of monocytosis. ${ }^{31-33}$ Based on the expression of CD14 and CD16, monocytes can be divided into three categories: $\mathrm{CD}^{+} 4^{+} / \mathrm{CD} 16^{-}$classical (M01), CD14 ${ }^{\text {low }} / \mathrm{CD} 6^{+}$intermediate (M02), and $\mathrm{CD} 14^{-} / \mathrm{CD}_{16}{ }^{+}$non-classical monocytes (M03) (Figure 2). ${ }^{31,32}$ These subsets differ in their chemokine receptor expression, phagocytic activity, epigenetic profiles and have unique metabolic pathway dependencies. ${ }^{32,34}$ In CMML, a pivotal study demonstrated an increase in the M01 subset, with an established cutoff $>94 \%$ being associated with sensitivity and specificity values of $90.6 \%$ and $95.1 \%$, respectively. ${ }^{32}$ These findings have been validated independently and this method importantly is effective in identifying MDS patients whose disease eventually evolves into CMML and in distinguishing CMML from MPN with monocytosis..$^{31,33,35,36}$ False negative findings secondary to autoimmunity/inflammation (expansion of the M02 fraction) and false positive findings in myeloid neoplasms such as MDS, atypical chronic myeloid leukemia and classical chronic myeloid leukemia have been documented. ${ }^{33,37,38}$ I do use this flow cytometry assay for screening patients who present with sustained monocytosis, especially when there is suspicion of an underlying clonal process and to differentiate CMML-associated monocytosis from other myeloid neoplasms with monocytosis. ${ }^{39}$

Next-generation sequencing assays are an important part of the diagnosis and prognostication of CMML. Virtually all CMML patients will have detectable somatic mutations involving genes regulating the epigenome, splicing, signaling and transcription. ${ }^{4,5,11,40}$ Clonal compositions help to define PCMML and dCMML subtypes and contribute towards AML transformation. ${ }^{10}$ Single-cell sequencing data have shown that TET2 mutations are usually the founder mutations occurring at the hematopoietic stem cell level. ${ }^{41}$ These mutations impact multipotent progenitor and common myeloid progenitor cells, skewing differentiation towards granulomonocytic progenitors and mature monocytes, respectively (Figure 1). ${ }^{41}$ Second-order mutations tend to accumulate in multipotent progenitor and common myeloid progenitor cells, often involving additional sites/alleles on TET2, spliceosome component genes (SRSF2, rarely SF3B1 and U2AF1) and additional epigenetic regulators ( $A S X L 1$, rarely $E Z H 2)^{11,41-43}$ Signaling mutations, such as NRAS, CBL, PTPN11, KRAS, NF1 and JAK2V671F, can sometimes be early/founder mutations, with inherent hypersensitivity of hematopoietic stem/progenitor cells to granulocyte-macrophage colony-stimulating factor (like the pCMML pediatric counterpart, juvenile myelomonocytic leukemia), but can also be later/subclonal events, giving rise to pCMML. ${ }^{10,41,44}$ Similarly, acquisition of additional epigenetic (DNMT3A, rarely IDH1/2) and splicing mutations (SF3B1) and mutations involving transcription factors (RUNX1) often gives rise to a dCMML phenotype. ${ }^{40,45}$ Somatic copy number alterations, especially copy neutral loss of heterozygosity, is common in CMML and frequently involves TET2 (4q24) and CBL (11q23), playing a role in clonal evolution..$^{10}$ Genetic alterations involving protein coding regions, including copy number gains and losses in driver mutations were only able to explain $44 \%$ of CMML cases that transformed to AML, indicating that mechanisms of

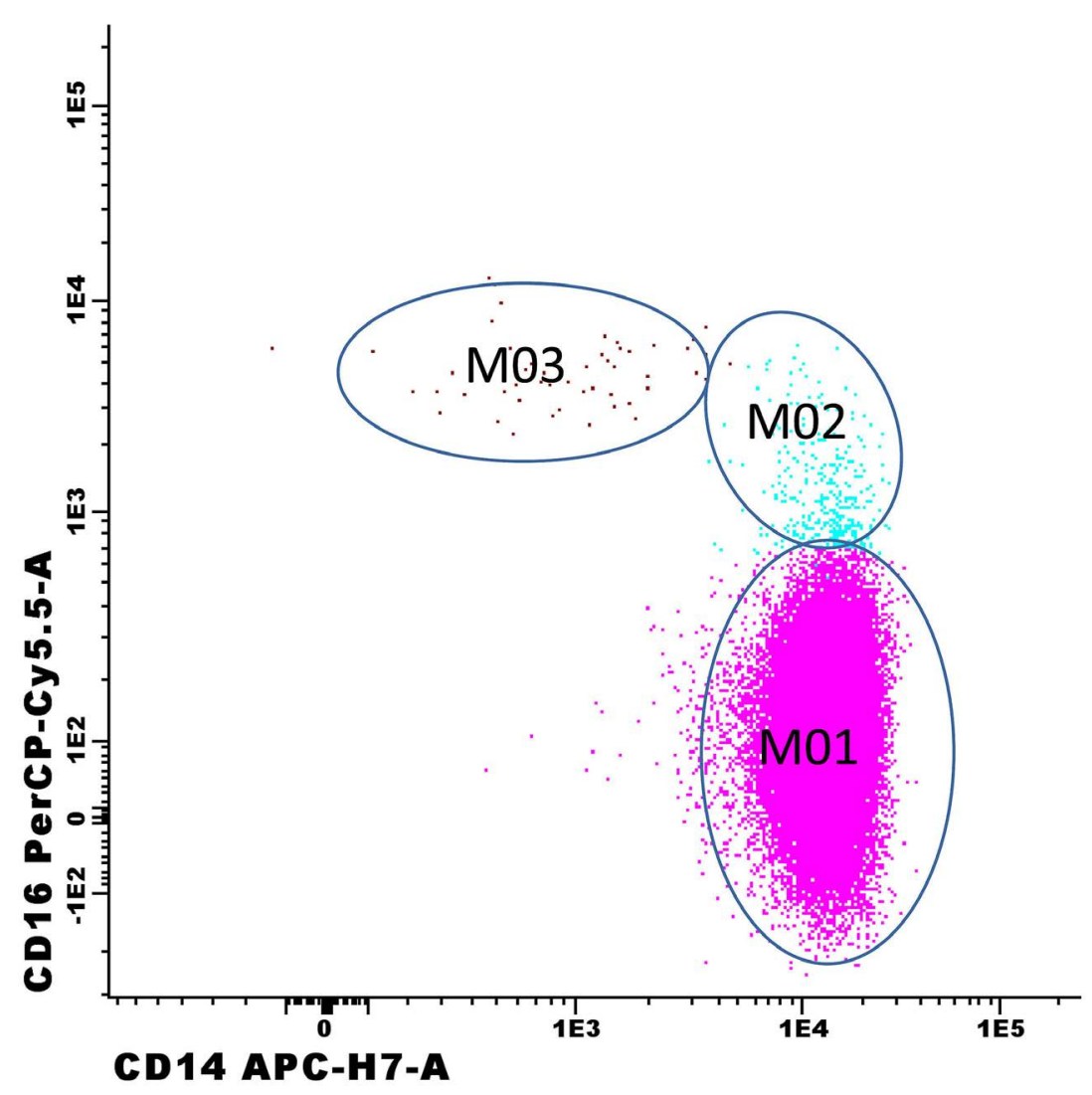

Figure 2. Flow cytometric analysis of monocyte repartitioning. Flow cytometry demonstrating a markedly expanded M01 $\left(\mathrm{CD} 14^{+} / \mathrm{CD} 16^{-}\right)$monocyte fraction, approximating $98.79 \%$, in a patient with chronic myelomonocytic leukemia. M01: classical monocytes; M02: intermediate monocytes; M03: non-classical monocytes. 
AML transformation remain to be elucidated. ${ }^{10}$ It is important to note that germline variants have been implicated in the pathogenesis of CMML, with cases having been documented in the context of germline mutations involving RUNX1, ANKRD26, ETV6, CHEK2, CDK2NA and GATA2. ${ }^{46,47}$ Recently, a $700 \mathrm{~kb}$ germline duplication localized to 14.q32.2, resulting in overexpression of $A T G 2 B$ and GSKIP genes, was described and has been associated with familial MPN and CMML. ${ }^{48}$ Based on family histories, age of onset and the heterozygous nature of variant allele fractions, I routinely assess germline DNA from extracted hair follicles/skin fibroblasts to assess for germline predisposition syndromes. ${ }^{49}$

In CMML, bone marrow biopsies are often hypercellular with granulocytic hyperplasia and mild to modest dysplasia (Figure 3D, E). Bone marrow monocytosis can be present, but is often difficult to appreciate and immunohistochemical studies that aid in the identification of monocytes and

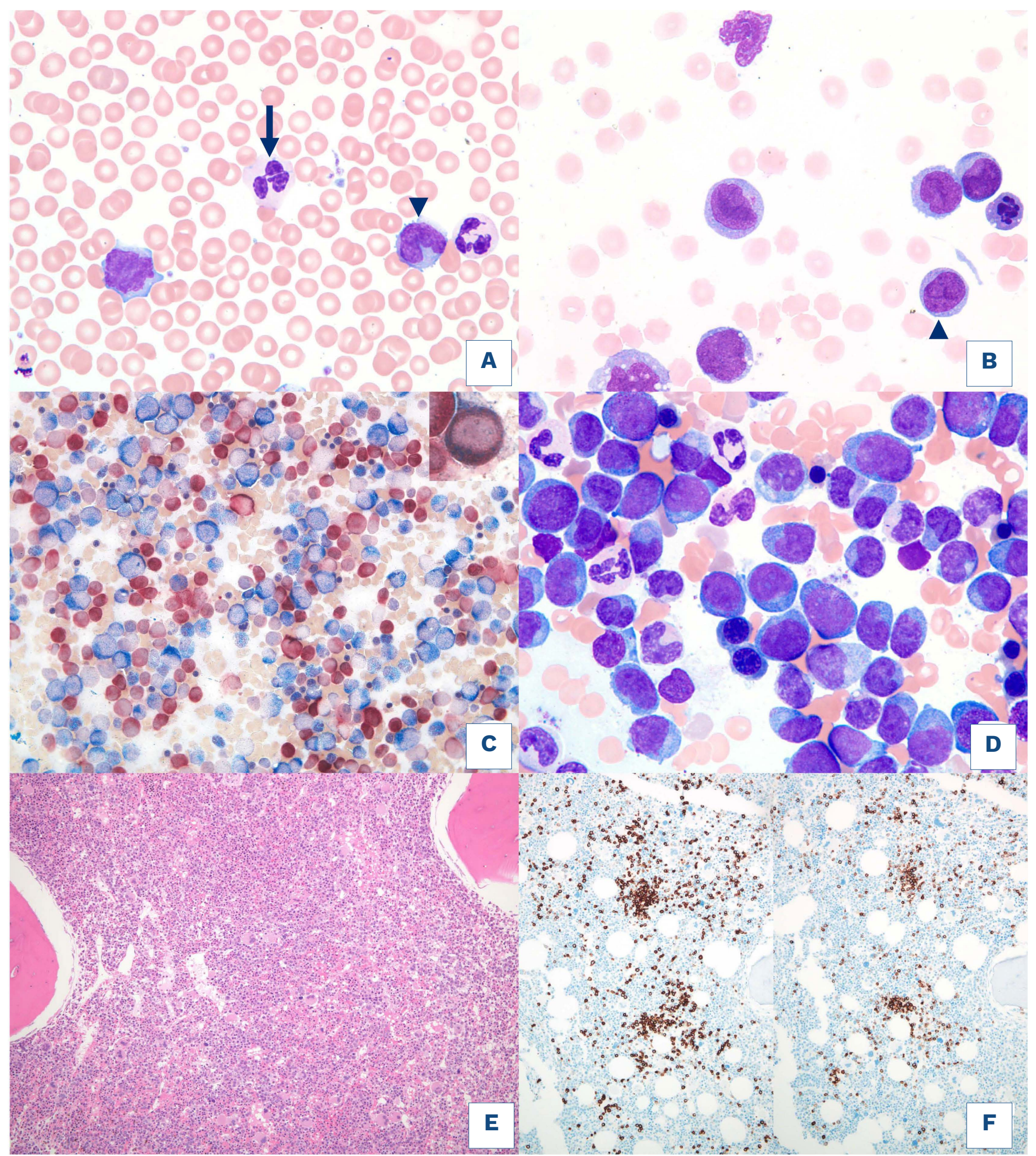

Figure 3. Peripheral blood and bone marrow findings in patients with chronic myelomonocytic leukemia. (A) Peripheral blood smear with hypogranular neutrophil (black arrow) and promonocytes (black arrowhead). (B) Bone marrow aspirate with promonocytes (black arrowhead). (C) Butyrate esterase/chloroacetate esterase stain demonstrates numerous butyrate esterase-positive monocytes, as well as dual esterase-positive cells (inset). (D) Bone marrow aspirate with increased blasts. (E) Bone marrow biopsy demonstrating a hypercellular bone marrow. (F) Small plasmacytoid dendritic cell nodules CD123+ (left) and $\mathrm{CD} 03^{+}$(right). 
their precursors are recommended (Figure 3C). ${ }^{50}$ Almost $80 \%$ of patients will demonstrate micro-megakaryocytes with abnormal nuclear contours and lobations, and $20-30 \%$ of patients can have an increase in reticulin fibrosis. ${ }^{50} \mathrm{Ap}-$ proximately $30 \%$ of patients demonstrate nodules composed of mature plasmacytoid dendritic cells that are

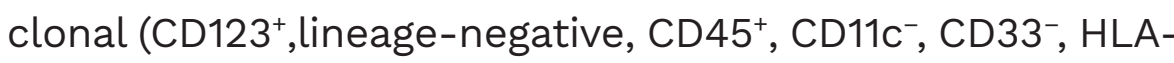
$\mathrm{DR}^{+}, \mathrm{BDCA}-2^{+}$and $\mathrm{BDCA}-4^{+}$), often have RAS pathway mutations and predict for an inferior AML-free survival (Figure $3 F) .{ }^{51}$ The identification of promonocytes requires expertise and these cells should be summated with blasts when estimating the blast count (Figure 3A, B) ${ }^{52}$ Promonocytes are described as monocytic precursors that have a delicately convoluted, folded or grooved nucleus with finely dispersed chromatin, a small indistinct or absent nucleolus, and finely granulated cytoplasm. ${ }^{52,53}$ On immunophenotyping the abnormal bone marrow cells often express myelomonocytic antigens such as CD13 and CD33, with variable expression of CD14, CD68 and CD64. Markers of aberrant expression include CD2, CD15, and CD56 or decreased expression of CD13, CD14, HLA-DR, CD64 or CD36. The presence of myeloblasts can often be detected by expression of CD34. The most reliable markers on immunohistochemistry include CD68R and CD163. On cytochemical analysis, monocytes are often positive for non-specific esterases and lysozyme, while the granulocytic precursors are often positive for lysozyme and chloroacetate esterase (Figure 3C). This technique can help to differentiate CMML from other overlap neoplasms in which bone marrow monocytosis is uncommon. Conventional karyotyping on the bone marrow is important for cytogenetic risk stratification, with approximately $70-80 \%$ of patients demonstrating a normal karyotype. Common abnormalities include +8 and $-Y$, with isolated del5q, complex and monosomal karyotypes being very uncommon (complex and monosomal karyotypes can be seen in patients with therapy-related CMML). ${ }^{16,54,55}$ The CMML-specific prognostic scoring system (CPSS) and the Mayo-French cytogenetic risk stratification system are two commonly used karyotype-based prognostic models for patients with CMML (Table 2). ${ }^{54,55}$

\section{How I approach chronic myelomonocytic leukemia variants and molecularly defined entities with monocytosis}

The diagnosis and management of CMML variants and molecularly defined entities presenting with monocytosis, mimicking CMML, require special attention. These variants can broadly be divided into three categories: (i) oligomonocytic CMML; (ii) CMML associated with a concomitant myeloid neoplasm; and (iii) molecularly defined entities with monocytosis.

Oligomonocytic CMML. This category encompasses patients who present with sustained relative monocytosis ( $\geq 10 \%$ of white blood cells) and absolute monocytosis not meeting current diagnostic criteria for CMML (absolute monocyte count $\left.0.5-<1.0 \times 10^{9} / \mathrm{L}\right) .{ }^{24}$ Based on the 2016 WHO classification, these patients would be classified as having either MDS or MDS/MPN-Unclassifiable. ${ }^{1}$ Except for an absolute monocyte count of $\geq 1 \times 10^{\%} / \mathrm{L}$, if these patients meet other CMML diagnostic criteria, along with a M01 fraction $>94 \%$ on monocyte repartitioning flow cytometry, and a molecular signature consistent with CMML (TET2, SRSF2, and ASXL1), I consider them as having oligomonocytic CMML and follow and manage them as such. Over time, several of these patients will have clonal evolution to either CMML or secondary AML.

CMML associated with a concomitant myeloid neoplasm. This category includes several variants, with the two most important being systemic mastocytosis (SM) with CMML (SM-CMML) and JAK2V617F-mutant $\mathrm{CMML}^{24} \mathrm{CMML}$ is the most frequent hematologic neoplasm associated with SM. I diagnose SM-CMML in patients who meet WHO criteria for both entities. In these patients, the SM component can present as either indolent or aggressive SM, with mast cell leukemia being extremely infrequent. ${ }^{56}$ In a Mayo Clinic study of 50 patients with SM-CMML, survival outcomes were similar to those of patients with CMML (24 months for SM-CMML vs. 18 months for CMML; $P=0.08$ ), There was a higher frequency of $K I T$ and $C B L$ mutations in SM-CMML and CMML-based prognostic models were not effective in risk stratification in this condition due to the confounding impact of SM. ${ }^{56}$ In CMML patients who have a detectable KITD816V mutation, a known driver oncogene in SM (>90\% of cases), I universally assess for concomitant SM clinically and by using laboratory techniques such as serum tryptase levels ( $>20 \mathrm{ng} / \mathrm{mL}$ ), bone marrow morphology, flow cytometry (aberrant expression of CD2 and/or CD25 on mast cells) and immunohistochemistry (CD117 and tryptase). For SM-CMML patients there are several exciting KIT-directed targeted therapies for the SM component (e.g., midostaurin and avapritinib) and given that in SMCMML, neoplastic monocytes are also often KIT mutated, ${ }^{57}$ these drugs can sometimes effectively decrease monocyte counts and infiltrative disease burden..$^{58}$ JAK2V617F-mutant CMML usually gives rise to a PCMML subtype with higher hemoglobin levels and absolute monocyte counts, with monocyte repartitioning by flow being a useful tool to distinguish this entity from MPN with monocytosis. ${ }^{59}$ On rare occasions, it becomes very difficult to distinguish the concomitant presence of a JAK2V617F MPN with bona fide CMML and in these instances, I manage each symptomatic component individually.

Molecularly defined entities with monocytosis. This category includes myeloid and lymphoid neoplasms pres- 
enting with monocytosis in the context of rearrangements involving PDGFRA, PDGFRB, FGFR1 and PCM1-JAK2. These entities are best designated by their molecular drivers and are currently not referred to as CMML. The recognition of these entities is very important given that they can present with a lymphoproliferative component and eosinophilia and can be targeted with tyrosine kinase inhibitors..$^{19,60}$

Table 2. Chronic myelomonocytic leukemia risk stratification models.

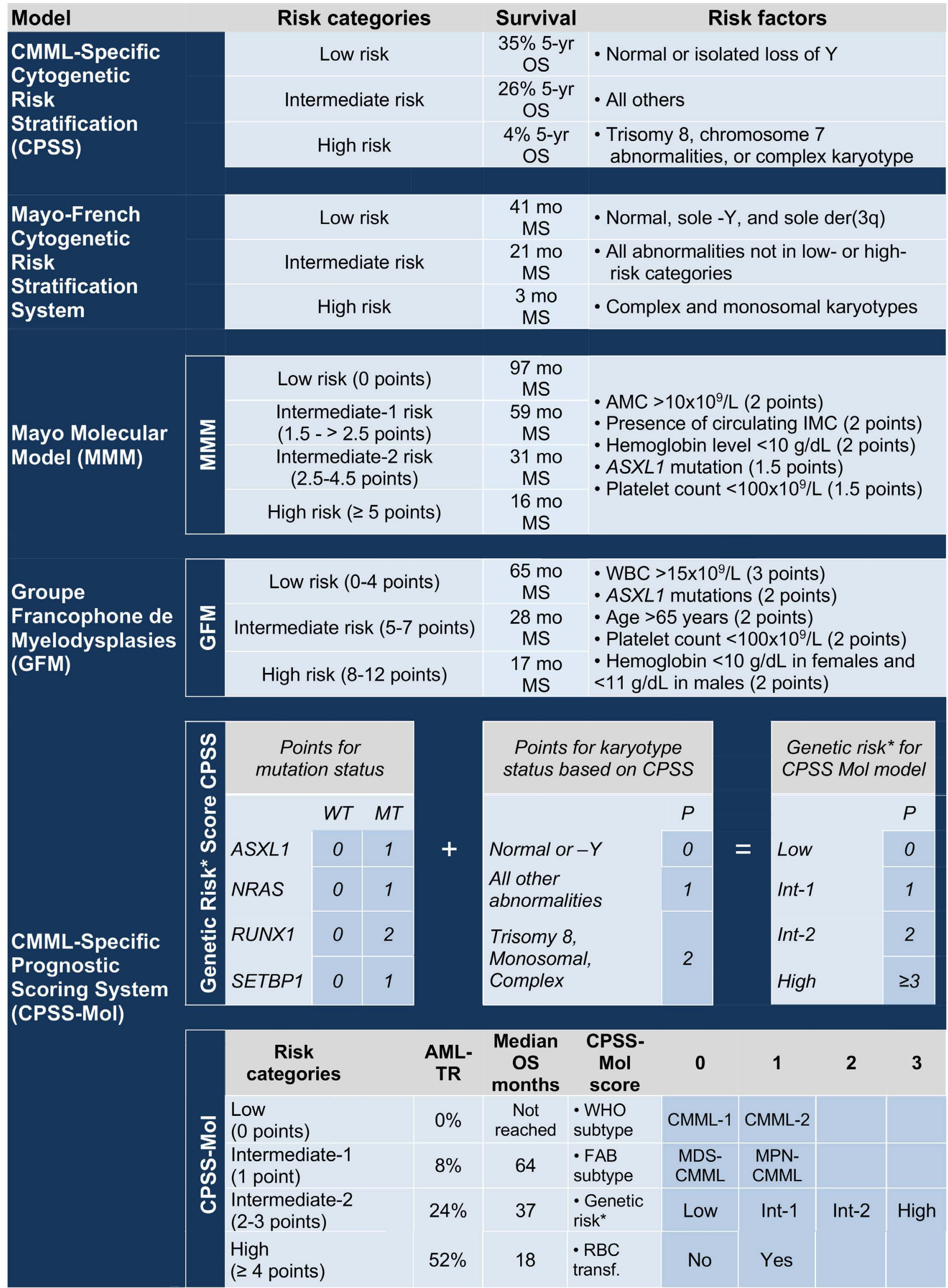

OS: overall survival; yr: year; mo: months; MS: median survival; AMC: absolute monocyte count, IMC: immature myeloid cells; WBC: white blood cell count; WHO_World Health Organization; FAB: French American and British classification; WT: wild type, MT: mutant, AML-TR: acute myeloid leukemia transformation rate, RBC transf: red blood cell transfusion; CMML: chronic myelomonocytic leukemia; MDS: myelodysplastic syndrome; MPN: myeloproliferative neoplasm. 


\section{How I prognosticate outcomes for patients with chronic myelomonocytic leukemia}

While there are numerous prognostic models for patients with CMML, models such as the Bournemouth, Lille, International Prognostic Scoring System (IPSS) and revisedIPSS scores are primarily designed for patients with MDS and excluded patients with PCMML. ${ }^{61,62}$ The MD Anderson prognostic system (MDAPS) is CMML-specific and identified a hemoglobin level $<12 \mathrm{~g} / \mathrm{dL}$, presence of circulating immature myeloid cells (myelocytes, promyelocytes and metamyelocytes), absolute lymphocyte count $>2.5 \times 10^{9} / \mathrm{L}$ and $\geq 10 \%$ bone marrow blasts as independent predictors for inferior survival. ${ }^{63}$ The Global MDAPS was then developed for patients with MDS, secondary MDS and CMML, with prognostic factors including; older age, poor performance status, thrombocytopenia, anemia, increased bone marrow blasts, leukocytosis (>20x10\%/L), chromosome 7 or complex cytogenetic abnormalities and a prior history of red blood cell transfusions. ${ }^{64}$ This model identified four prognostic groups with median survivals of 54 (low), 25 (intermediate-1), 14 (intermediate-2) and 6 months (high), respectively. ${ }^{64}$ The CPSS model was developed in Europe and identified PCMML versus dCMML subtypes, WHO CMML-subtypes, red blood cell transfusion dependency and the CPSS cytogenetic risk stratification system as being prognostic for survival. ${ }^{54,65}$ The Mayo prognostic model identified hemoglobin $<10 \mathrm{~g} / \mathrm{dL}$, platelet count $<100 \times 10^{9} / \mathrm{L}$, absolute monocyte count $>10 \times 10^{9} / \mathrm{L}$ and circulating immature myeloid cells as being independently prognostic. ${ }^{6}$

The discovery of somatic mutations in CMML resulted in the development of contemporary molecular prognostic models. The Group Francophone des Myelodysplasies (GFM) demonstrated an adverse prognostic effect for truncating ASXL1 mutations in 312 patients with CMML; additional risk factors included age $>65$ years, white blood cell count $>15 \times 10^{9} / \mathrm{L}$, platelet count $<100 \times 10^{9} / \mathrm{L}$ and hemoglobin $<10 \mathrm{~g} / \mathrm{dL}$ in females and $<11 \mathrm{~g} / \mathrm{dL}$ in males. ${ }^{5}$ The GFM model assigns three adverse points for white blood cell count $>15 \times 10^{9} / L$ and two adverse points for each one of the remaining risk factors, resulting in a three-tiered risk stratification; low (0-4 points), intermediate (5-7) and high (8-12), with respective median survivals of $56,27.4$ and 9.2 months. ${ }^{5}$ To further clarify the prognostic relevance of $A S X L 1$ mutations, the Mayo Molecular Model (MMM) was developed as a collaborative effort between the GFM and Mayo Clinic $(\mathrm{n}=466) .{ }^{66}$ Adverse prognostic factors included truncating $A S X L 1$ mutations, absolute monocyte count $>10 \times 10^{9} / \mathrm{L}$, hemoglobin $<10 \mathrm{~g} / \mathrm{dL}$, platelets $<100 \times 10^{9} / \mathrm{L}$ and circulating immature myeloid cells. Based on these variables a regression coefficient-based prognostic model was developed with the following risk cat- egories; high ( $\geq 3$ risk factors), intermediate-2 (2 risk factors), intermediate-1 (1 risk factor), and low (no risk factors) risk, with median survivals of 16, 31, 59 and 97 months, respectively. ${ }^{67}$ The CPSS model was also updated to include gene mutations involving ASXL1, RUNX1, NRAS and SETBP1 (CPSS-Mol). ${ }^{4}$ Gene mutations along with karyotypic abnormalities are used to calculate the CPSS genetic score. One point each is assigned for an intermediate- 1 genetic score, white cell count $\geq 13 \times 10^{9} / \mathrm{L}$, bone marrow blasts $\geq 5 \%$ and red blood cell transfusion dependency, two points for intermediate- 2 genetic score and three points for a high risk genetic score. ${ }^{4}$ The CPSS-Mol stratifies patients into four categories, low (0 risk factors), intermediate-1 (1 risk factor), intermediate-2 (2-3 risk factors) and high ( $\geq 4$ risk factors) risk, with median overall survival not reached, 64, 37 and 18 months; with 4-year leukemic transformation rates of $0 \%, 3 \%, 21 \%$ and $48 \%$, respectively. ${ }^{4}$ Table 2 highlights relevant CMML-specific prognostic models along with their component variables. Recently a CMML transplant model was developed (CMML transplant score) which assigned four points for the presence of ASXL1 and/or NRAS mutations, four points for bone marrow blasts $>2 \%$ and one point for each hematopoietic stem cell transplant (HSCT) comorbidity index, effectively stratifying for both overall survival and non-relapse mortality. ${ }^{68}$

In practice, any of the three molecularly integrated CMML prognostic models can be used for risk stratification. While these models have not been formally compared against each other, they share several overlapping prognostic features, especially anemia, elevated white blood cell counts and truncating $A S X L 1$ mutations. ${ }^{4,5,11}$ I use both the MMM and the CPSS-Mol model for risk stratification at my institution.

\section{How I manage patients with chronic myelomonocytic leukemia}

The first step in the management of CMML patients is establishing an accurate diagnosis, followed by personalized risk stratification. Using any one of the molecularly integrated prognostic models, CMML patients can be stratified into lower risk and higher risk groups (Figure 4). Management strategies for these two risk groups are described below.

\section{Lower-risk chronic myelomonocytic leukemia}

I define lower-risk CMML patients as those who fall into low and intermediate-1 risk categories based on the MMM and the CPSS-Mol, or the low-risk category of the GFM model. On average these patients have a median overall survival of 60-100 months 4,5,11 $^{4}$ and the following treatment strategies can be adopted for their care: 


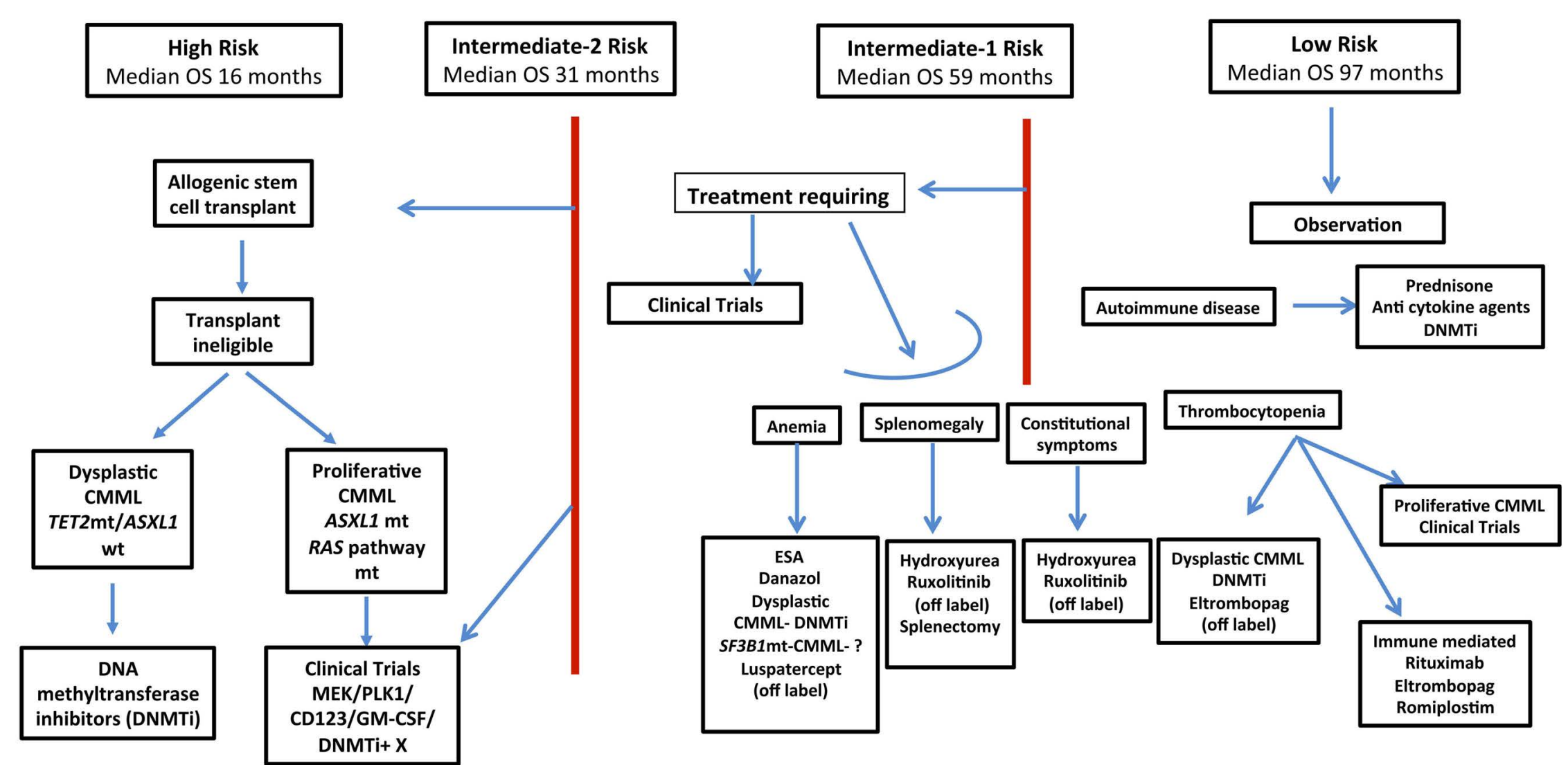

Figure 4. Management algorithm for chronic myelomonocytic leukemia based on risk stratification using the Mayo Molecular Model (Patnaik MM et al. Leukemia 2014). CMML: chronic myelomonocytic leukemia, DNMTi: DNA methyltransferase inhibitors, X: new clinical trial investigational agent.

Observation and supportive care. Several low-risk patients can be observed without any CMML-directed therapies, with serial blood count measurements and management of symptoms as needed. There are some data to suggest that in PCMML, permissive leukocytosis/monocytosis can be associated with increased lysozyme levels and a higher prevalence of chronic kidney disease (lysozyme nephropathy), and in select asymptomatic pCMML patients with white blood cell counts $>30 \times 10^{9} / \mathrm{L}$, I do recommend hydroxyurea to control blood counts and proliferative features. ${ }^{69}$ Side effects associated with hydroxyurea include nausea, vomiting, diarrhea, fever, mouth sores, photosensitivity, myelosuppression, and chronic non-healing leg/ankle ulcers.

Anemia. Ineffective erythropoiesis contributes to significant morbidity and mortality in CMML. While there are limited CMML-specific prospective data for anemia management, akin to MDS, the management of anemia largely centers around the use of red blood cell transfusions and erythropoiesis-stimulating agents. Extrapolating from MDS-based trials, in CMML, erythropoiesis-stimulating agents are also more likely to be effective in lower-risk patients, especially those with endogenous erythropoietin levels $<200 \mathrm{U} / \mathrm{L}$ and those with low or no dependency on red blood cell transfusions (40-70\% response rates). ${ }^{70-72}$ The median duration of response to erythropoiesis-stimulating agents is 12-18 months, with limited options after progression. I usually use fixed doses of recombinant human erythropoietin or darbepoetin and strictly avoid the use of granulocyte colony-stimulating factor) given the higher baseline risk of splenic rupture in CMML patients. ${ }^{73}$ I closely monitor for adverse vascular side effects associated with erythropoiesis-stimulating agents, such as treatment-emergent hypertension and thromboembolism and do not administer these agents when hemoglobin levels are $>11 \mathrm{~g} / \mathrm{dL}$. Luspatercept is a recombinant fusion protein that traps GDF 11 and activin ligands belonging to the TGF- $\beta$ superfamily, decreasing SMAD2 and SMAD3 signaling, enabling latestage erythroid maturation and has been approved by the Food and Drug Administration for patients with $\beta$-thalassemia and MDS-ring sideroblasts. ${ }^{74}$ While there are no clear safety or efficacy data on the use of luspatercept in CMML, I do consider off-label use in a select group of SF3B1-mutant CMML patients with bone marrow-ring sideroblasts, who are ineligible for erythropoiesis-stimulating agents or in whom these agents have failed. ${ }^{43}$ Luspatercept is in general well tolerated with side effects including headaches, bone pain, arthralgia and fatigue. We have recently defined SF3B1mutant CMML as a CMML subtype with predominant dysplastic features, with a low frequency of ASXL1 mutations, higher frequency of JAK2V61F mutations, concurrent splicing mutations, and a superior AML-free survival. ${ }^{43}$ Other options for anemia management include danazol (an anabolic steroid), lenalidomide (an immunomodulatory agent; note - isolated del5q is seen in $<1 \%$ of CMML cases) and DNA methyltransferase (DNMT) inhibitors, such as 5-azacitidine, decitabine and oral decitabine combined with cedazuridine (cytidine deaminase inhibitor), given in either conventional doses, or in attenuated dose schedules. ${ }^{75,76}$ Given that these strategies for managing anemia are either suboptimal or not durable, I strongly encourage participation in clinical trials. 
Splenomegaly. Symptomatic splenomegaly can be a significant issue in patients with PCMML. Clinical issues related to splenomegaly include early satiety, abdominal pain and tenderness, constitutional symptoms, referred shoulder pain, hiccoughs and mechanical obstruction of abdominal organs. ${ }^{77}$ Splenic infarction and spontaneous splenic rupture can result in abdominal catastrophes. ${ }^{73}$ । usually manage symptomatic splenomegaly, or massive splenomegaly, with cytoreductive therapy, with hydroxyurea being my first choice. There are recent encouraging data on the use of ruxolitinib, a JAK1/2 inhibitor, in patients with CMML, with $43 \%$ of CMML patients with baseline splenomegaly demonstrating a spleen response. ${ }^{78}$ Ruxolitinib was well tolerated with the two most common grade 3 and 4 treatment-related toxicities being anemia (10\%) and thrombocytopenia (6\%). I have used ruxolitinib off-label in select patients with good effect. DNMT inhibitors, splenic radiation and splenectomy are generally avoided, given inherent complications such as worsening cytopenias with DNMT inhibition, lack of durable responses with radiation and surgical morbidity/mortality associated with splenectomy.

Thrombocytopenia. Thrombocytopenia in CMML has diverse etiologies including splenic sequestration from hypersplenism, immune-mediated thrombocytopenia and bone marrow failure from progressive disease. Autoimmune phenomena, including immune-mediated thrombocytopenia, can be seen in $20-30 \%$ of CMML patients. ${ }^{79,80}$ While corticosteroids and rituximab have been used for patients with suspected immune-mediated thrombocytopenia, the use of thrombopoietin analogs, especially eltrombopag in CMML needs caution. There are reports of PCMML patients demonstrating worsening proliferative features, circulating blasts and bone marrow fibrosis on exposure to eltrombopag. ${ }^{81}$ The GFM however has completed a yet to be published phase II trial (NCT02323178) assessing the safety of eltrombopag in CMML patients with severe thrombocytopenia (platelet count $<50 \times 10^{9} / \mathrm{L}$ ). In this study eltrombopag was relatively well tolerated (median dose $150 \mathrm{mg}$; range, $100-300 \mathrm{mg}$ ), with $46.7 \%$ of patients achieving a platelet response (10 with dCMML and 4 with $\mathrm{pCMML}$ ) that in general was not durable (median duration 3.4 months; range, 1.7-11.6 months). I use extreme caution when prescribing eltrombopag for $\mathrm{PCMML}$ patients with proliferative features. Other options for thrombocytopenia include splenectomy when immunemediated thrombocytopenia or splenic sequestration is suspected and DNMT inhibitors if the etiology is diseaserelated bone marrow dysfunction/failure.

Autoimmune manifestations. Autoimmune and systemic inflammatory manifestations such as erythema nodosum, leukocytoclastic vasculitis, Sweet syndrome, polymyalgia rheumatica, seronegative arthritis, and mixed connective tissue disorder-like syndromes can be seen in $20-30 \%$ of patients, with manifestations often preceding the diagnosis of CMML. ${ }^{79,80}$ With growing evidence on the role played by clonal hematopoiesis-clones in amplifying inflammation and endothelial dysfunction, there is more understanding on the pathobiology of inflammation and autoimmunity in CMML. ${ }^{82-84}$ Cytokines whose levels are elevated in CMML patients include, IL-8, IP-10, IL-1RA, TNF$\alpha, I L-6, M C P-1 / C C L 2, H G F, M-C S F, V E G F, I L-4$, and IL-2RA, with decreased levels of IL-10 being associated with adverse prognosis. ${ }^{85}$ The transcriptional signature of CMML monocytes is also highly inflammatory, with upregulation of multiple inflammatory pathways, including TNF- $\alpha$, IL- 6 and IL-17. ${ }^{86}$ While corticosteroids and steroid-sparing/disease-modifying agents are often used in the management of these symptoms, I use DNMT inhibitors in conventional or low doses, for more durable responses. ${ }^{80}$ Azathioprine is a steroid-sparing immunosuppressive agent that I strictly avoid, given its strong association with therapyrelated myeloid neoplasms.

\section{Higher-risk chronic myelomonocytic leukemia}

I define higher-risk CMML patients as those who fall into intermediate-2 and high-risk categories based on the MMM and the CPSS-Mol, or the high-risk category of the GFM model. On average these patients have a median overall survival $<2$ years ${ }^{4,5,11}$ and the following treatment strategies can be adopted for their care.

Allogeneic HSCT. Allogeneic HSCT remains the only potentially curative option for patients with CMML. However, given the older age at presentation and associated morbidities, most patients are not eligible. ${ }^{87}$ At our institution, CMML patients with higher-risk disease diagnosed $<75$ years of age and with an acceptable HSCT-comorbidity index (deemed by an expert committee) are usually referred for allogeneic HSCT. ${ }^{88}$ A recent consensus document from an expert panel does recommend upfront allogeneic HSCT for intermediate- 2 and high-risk CMML patients (CPSS risk stratification), with $<10 \%$ bone marrow blasts. ${ }^{89}$ For patients with $>10 \%$ bone marrow blasts, I usually cytoreduce with DNMT inhibition or AML-like induction therapy, especially in "younger and fit" patients. ${ }^{8,88}$ In the largest study to date, the European Group for Blood and Bone Marrow Transplant reported outcomes of 513 patients (median age 53 years) of whom 249 received myeloablative conditioning and 228 received reduced intensity conditioning. ${ }^{90}$ The 4-year nonrelapse mortality was $41 \%$ and relapse rate $32 \%$, accounting for a 4 -year relapse-free survival rate of $27 \%$ and an overall survival rate of $33 \% .{ }^{90}$ In this study the only factor prognostic for favorable outcomes was the achievement of a complete remission prior to HSCT. Data with regard to the use of DNMT inhibitors prior to HSCT in CMML are largely retrospective and somewhat controversial, given their lack of disease-modifying efficacy and propensity to worsen existing cytopenias. ${ }^{91}$ In general, I try to avoid DNMT in- 

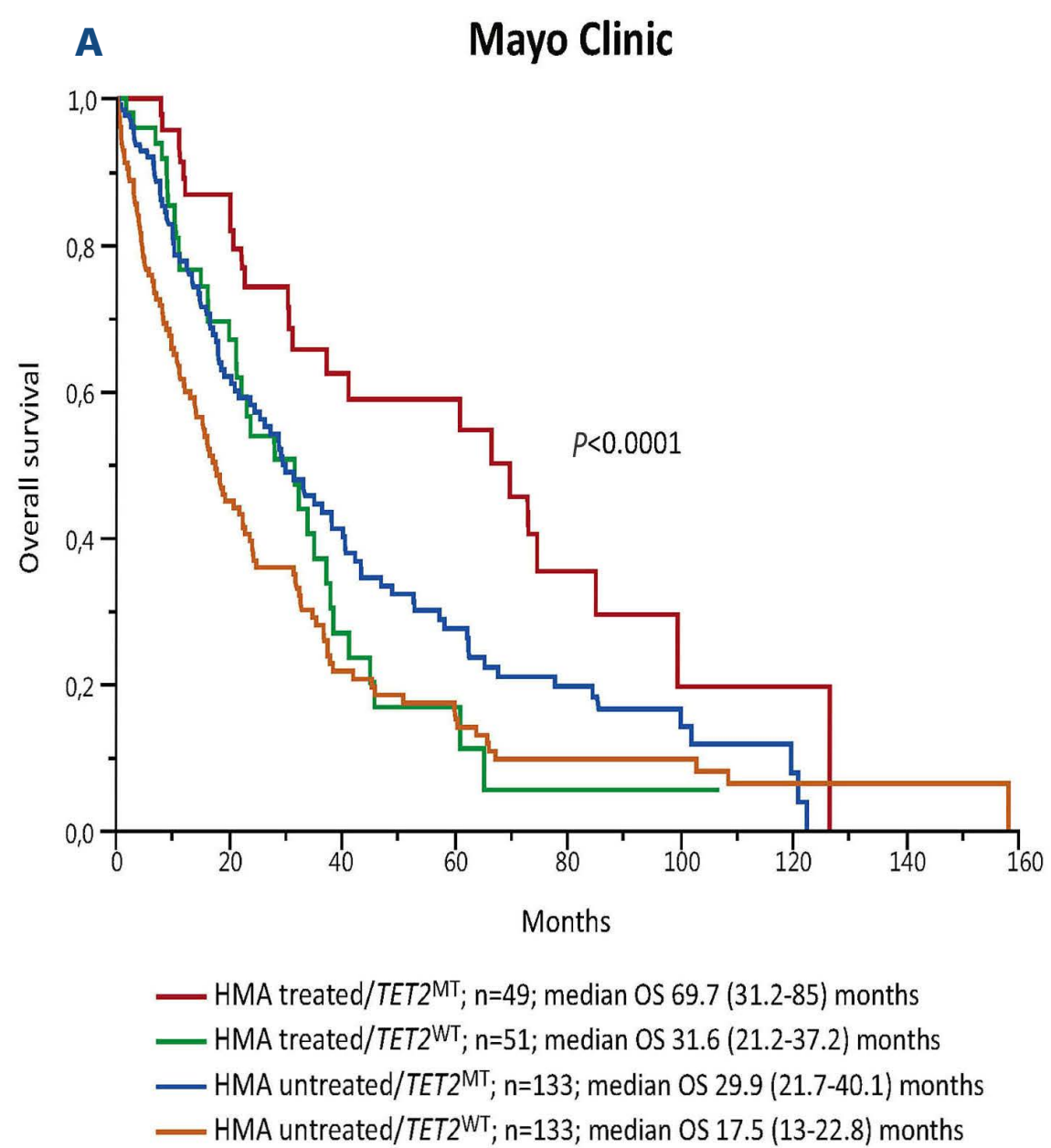

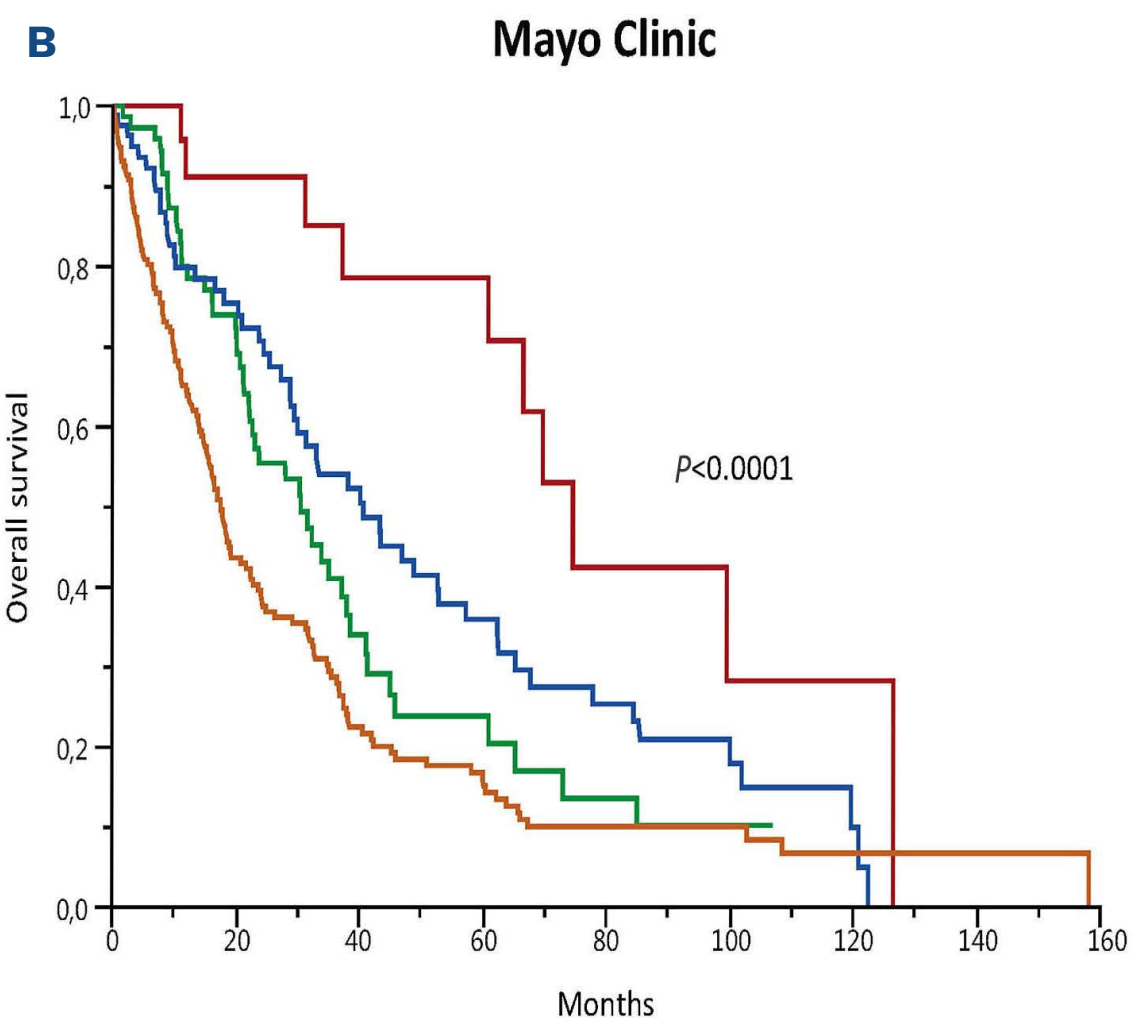

— HMA treated/ASXL1 ${ }^{\text {WT }} /$ TET2 $^{\mathrm{MT}}$; $\mathrm{n}=25$; median OS 74.6 (60.9-126.5) months

— HMA treated/ASXL1 ${ }^{\mathrm{MT}}$ \& ASXL1 ${ }^{\mathrm{WT}} / \mathrm{TET}^{\mathrm{WT}}$; $\mathrm{n}=75$; median OS 30.5 (22.1-38) months

— HMA untreated/ASXL1 ${ }^{\text {WT }} /$ TET2 $^{\mathrm{MT}}$; $\mathrm{n}=83$; median OS 40.7 (28.9-52.9) months

— HMA untreated/ASXL1 ${ }^{\text {MT }}$ \& ASXL1 ${ }^{\text {WT }} /$ TET2WT; $^{\text {W }} \mathrm{n}=182$; median OS 17.6 (15-21.7) months

Figure 5. Impact of mutations on survival of patients stratified according to treatment with DNA methyltransferase inhibitors. (A, B) Kaplan-Meier curves demonstrating the impact of TET2 mutations (A) and the TET2mutant/ASXL1wildtype genotype (B) on survival outcomes, stratified by treatment or no treatment with DNA methyltransferase inhibitors (HMA: hypomethylating agents) in the Mayo Clinic CMML cohort. OS: overall survival.

hibition prior to HSCT, unless it is needed to cytoreduce a patient prior to conditioning therapy. A recent Mayo Clinic study confirmed the survival benefit offered by allogeneic HSCT in higher-risk CMML patients, with 5-year overall survival rates of $51 \%$ for patients with chronic phase CMML and $19 \%$ for those with blast transformed CMML. ${ }^{88}$ Soberingly the graft-versus-host disease relapse-free survival in the chronic phase cohort was only 7 months. With a greater availability of donor sources, alternative donor transplant strategies and an improving arsenal for management of graft-versus-host disease, we hope that allogeneic HSCT becomes a viable option for a greater number of patients. DNMT inhibitors. DNMT inhibitors such as 5-azacitidine, decitabine and the oral combination of decitabine with cedazuridine are the only drugs approved for the management of CMML by the U.S Food and Drug Administration. In Europe, 5-azacitidine remains the only drug approved for the management of CMML ( $>10 \%$ blasts). The approval of these agents was largely based on MDS predominant trials that included a small number of CMML patients, all of whom had a white blood cell count $<12 \times 10^{\%} / L$ (dCMML). ${ }^{92,93}$ The overall response rates to DNMT inhibitors are approximately $40-50 \%$, with true complete remission rates of $<20 \% .{ }^{94,95}$ In a seminal study, elaborate sequencing data demonstrated that DNMT inhibitors induce responses in CMML patients by epigenetically restoring normal hematopoiesis, without impacting mutational allele burdens, with disease progression to AML remaining inevitable. ${ }^{96}$ The struggle with using DNMT inhibitors in CMML is the lack of predictors or biomarkers of response. In a large multi-institutional study, we identified the presence of TET2 mutations in the absence of ASXL1 mutations being the best markers of response to DNMT inhibitors (Figure 5), similarly to the situation in MDS. ${ }^{97-99}$ In a recent prospective randomized trial assessing the efficacy of decitabine versus hydroxyurea in higher-risk PCMML patients $(n=170)$, there was no difference in overall survival or event-free survival between the hydroxyurea and decitabine arms (NCT02214407; Itzykson et al. ASH 2020). Adverse effects of DNMT inhibitors include nausea, vomiting, diarrhea, fatigue, and myelosuppression. I proactively educate my patients about these adverse effects and optimize supportive care for the preemptive management of the same.

I largely use DNTM inhibitors in dCMML subtypes with higher risk disease or clinically significant cytopenias, especially if they are TET2 mutant and ASXL1 wildtype (Figure 5). I avoid using DNMT inhibtors in PCMML patients with dominant RAS pathway mutations or with highly proliferative features. For these patients I proactively seek clinical trials with novel therapeutic agents. For patients who respond to DNMT inhibition, the median duration of re- 
sponse is $12-18$ months and survival after progression is dismal, ranging between 6-9 months. ${ }^{94}$ Given recent data suggesting improved survival rates in AML patients treated in combination with azacitidine and the bcl-2 inhibitor venetoclax, there has been interest in assessing this combination in CMML. ${ }^{100}$ Preclinical BH3 mimetic profiling data in CMML suggest that the malignant monocytes are addicted to mcl1 instead of bcl2 and that combination therapy with mcl1 and MAPK inhibitors might be a successful treatment strategy. ${ }^{101}$ While prospective data are awaited, a small retrospective series including CMML patients with blast transformation demonstrated suboptimal response rates (overall response rate - 50\% for CMML), with no significant difference from response rates seen with DNMT inhibitors alone..$^{102}$

Clinical trials. I actively seek out clinical trials for all our CMML patients, given the suboptimal response rates to conventional strategies. We have successfully carried out CMML-specific clinical trials and urge the scientific community to stop grouping CMML with MDS or MPN, given the unique biology of CMML. CMML-specific trials that have been completed or are currently accruing include trials assessing the safety and efficacy of lenzilumab (an anti-GM-CSF monoclonal antibody: NCT02546284), tipifarnib (a farnesyl transferase inhibitor: NCT02807272), ruxolitinib (a JAK1/2 inhibitor: NCT03722407), cobimetinib (a MEK inhibition: NCT04409639) and tagraxofusp (a protein conjugate involving IL-3 and truncated components of the diphtheria toxin: NCT02268253) in patients with CMML. ${ }^{103-}$ ${ }^{105}$ Preclinical data from our laboratory have demonstrated a unique RAS-KMT2A-PLK1 axis defining the PCMML phenotype, with PLK1 inhibition with the oral, selective PLK1 inhibitor onvansertib demonstrating excellent preclinical activity. ${ }^{10,106}$

\section{Conclusions}

CMML is a unique MDS/MPN overlap neoplasm with relative genetic homogeneity, but with marked clinical heterogeneity. The disease is seen in the elderly and frequently develops on the background of clonal hematopoiesis, with recurrent somatic mutations involving TET2, ASXL1, SRSF2 and the RAS pathway defining dysplastic and proliferative subtypes of CMML. For several years CMML was considered as a subtype of MDS, but from 2002 onwards, CMML has been rightfully recognized as a unique neoplasm with the development of CMML-specific prognostic models, response criteria, preclinical models and, most importantly, clinical trials; heralding a new future for this disease and affected patients. Several challenges remain, including the lack of uniform consensus on personalized prognostication and more importantly, the identification of disease-modifying targets and therapies that might ameliorate disease progression, improve quality of life, and potentially offer a cure.

\section{Disclosures}

My institution has received research funding from Stem Line therapeutics and Kura Oncology.

\section{Acknowledgments}

I would like to acknowledge all the CMML patients who have entrusted their lives and care in my hands; my institution and mentors for supporting me and my national and international collaborators and sponsors. I would also like to acknowledge DRS, and Matthew Howard and Michael Timm from the Department of Laboratory Medicine and Pathology for helping with blood and bone marrow images and flow cytometry data, respectively.

\section{References}

1. Arber DA, Orazi A, Hasserjian R, et al. The 2016 revision to the World Health Organization classification of myeloid neoplasms and acute leukemia. Blood. 2016;127(20):2391-2405.

2. Patnaik MM, Lasho T. Myelodysplastic syndrome/myeloproliferative neoplasm overlap syndromes: a focused review. Hematology Am Soc Hematol Educ Program. 2020;2020(1):460-464.

3. Patnaik MM, Lasho TL. Genomics of myelodysplastic syndrome/myeloproliferative neoplasm overlap syndromes. Hematology Am Soc Hematol Educ Program. 2020;2020(1):450-459.

4. Elena C, Galli A, Such E, et al. Integrating clinical features and genetic lesions in the risk assessment of patients with chronic myelomonocytic leukemia. Blood. 2016;128(10):1408-1417.

5. Itzykson R, Kosmider O, Renneville A, et al. Prognostic score including gene mutations in chronic myelomonocytic leukemia. $J$ Clin Oncol. 2013;31(19):2428-2436.

6. Patnaik MM, Padron E, LaBorde RR, et al. Mayo prognostic model for WHO-defined chronic myelomonocytic leukemia:
ASXL1 and spliceosome component mutations and outcomes. Leukemia. 2013;27(7):1504-1510.

7. Rollison DE, Howlader N, Smith MT, et al. Epidemiology of myelodysplastic syndromes and chronic myeloproliferative disorders in the United States, 2001-2004, using data from the NAACCR and SEER programs. Blood. 2008;112(1):45-52.

8. Patnaik MM, Wassie EA, Padron E, et al. Chronic myelomonocytic leukemia in younger patients: molecular and cytogenetic predictors of survival and treatment outcome. Blood Cancer J. 2015;5(2):e280.

9. Ball M, List AF, Padron E. When clinical heterogeneity exceeds genetic heterogeneity: thinking outside the genomic box in chronic myelomonocytic leukemia. Blood. 2016;128(20):2381-2387.

10. Carr RM, Vorobyev D, Lasho T, et al. RAS mutations drive proliferative chronic myelomonocytic leukemia via a KMT2APLK1 axis. Nat Commun. 2021;12(1):2901.

11. Patnaik MM, Itzykson R, Lasho TL, et al. ASXL1 and SETBP1 mutations and their prognostic contribution in chronic 
myelomonocytic leukemia: a two-center study of 466 patients. Leukemia. 2014;28(11):2206-2212.

12. Savona MR, Malcovati L, Komrokji R, et al. An international consortium proposal of uniform response criteria for myelodysplastic/myeloproliferative neoplasms (MDS/MPN) in adults. Blood. 2015;125(12):1857-1865.

13. Mason CC, Khorashad JS, Tantravahi SK, et al. Age-related mutations and chronic myelomonocytic leukemia. Leukemia. 2016;30(4):906-913.

14. You X, Liu F, Binder M, et al. Asxl1 loss cooperates with oncogenic Nras in mice to reprogram immune microenvironment and drive leukemic transformation. Blood. 2022;139(7):1066-1079.

15. Bernard E, Nannya Y, Hasserjian RP, et al. Implications of TP53 allelic state for genome stability, clinical presentation and outcomes in myelodysplastic syndromes. Nat Med. 2020;26(10):1549-1556.

16. Patnaik MM, Vallapureddy R, Yalniz FF, et al. Therapy relatedchronic myelomonocytic leukemia (CMML): molecular, cytogenetic, and clinical distinctions from de novo CMML. Am J Hematol. 2018;93(1):65-73.

17. Ohtake S. Chronic myelogenous leukemia with p190BCR-ABL expression: the missing link with monocytosis. Intern Med. 2002;41(12):1092-1093.

18. Patnaik MM, Lasho TL, Finke CM, Pardanani A, Tefferi A. Targeted next generation sequencing of PDGFRB rearranged myeloid neoplasms with monocytosis. Am J Hematol. 2016;91(3):E12-14.

19. Pardanani A, Ketterling RP, Li CY, et al. FIP1L1-PDGFRA in eosinophilic disorders: prevalence in routine clinical practice, long-term experience with imatinib therapy, and a critical review of the literature. Leuk Res. 2006;30(8):965-970.

20. Apperley JF, Gardembas M, Melo JV, et al. Response to imatinib mesylate in patients with chronic myeloproliferative diseases with rearrangements of the platelet-derived growth factor receptor beta. N Engl J Med. 2002;347(7):481-487.

21. Golub TR, Barker GF, Lovett M, Gilliland DG. Fusion of PDGF receptor beta to a novel ets-like gene, tel, in chronic myelomonocytic leukemia with $\mathrm{t}(5 ; 12)$ chromosomal translocation. Cell. 1994;77(2):307-316.

22. Tefferi A, Shah S, Mudireddy M, et al. Monocytosis is a powerful and independent predictor of inferior survival in primary myelofibrosis. Br J Haematol. 2018;183(5):835-838.

23. Patnaik MM, Timm MM, Vallapureddy R, et al. Flow cytometry based monocyte subset analysis accurately distinguishes chronic myelomonocytic leukemia from myeloproliferative neoplasms with associated monocytosis. Blood Cancer J. 2017;7(7):e584.

24. Valent P, Orazi A, Savona MR, et al. Proposed diagnostic criteria for classical chronic myelomonocytic leukemia (CMML), CMML variants and pre-CMML conditions. Haematologica. 2019;104(10):1935-1949.

25. Patnaik MM, Pophali PA, Lasho TL, et al. Clinical correlates, prognostic impact and survival outcomes in chronic myelomonocytic leukemia patients with the JAK2V617F mutation. Haematologica. 2019;104(6):e236-e239.

26. Daver N, Strati P, Jabbour E, et al. FLT3 mutations in myelodysplastic syndrome and chronic myelomonocytic leukemia. Am J Hematol. 2013;88(1):56-59.

27. Vallapureddy R, Lasho TL, Hoversten K, et al. Nucleophosmin 1 (NPM1) mutations in chronic myelomonocytic leukemia and their prognostic relevance. Am J Hematol. 2017;92(10):E614-E618.

28. Patnaik MM, Tefferi A. Chronic myelomonocytic leukemia: 2020 update on diagnosis, risk stratification and management. Am J
Hematol. 2020;95(1):97-115.

29. Kiss M, Caro AA, Raes G, Laoui D. Systemic reprogramming of monocytes in cancer. Front Oncol. 2020;10:1399.

30. Shen Q, Ouyang J, Tang G, et al. Flow cytometry immunophenotypic findings in chronic myelomonocytic leukemia and its utility in monitoring treatment response. Eur $\mathrm{J}$ Haematol. 2015;95(2):168-176.

31. Patnaik MM, Timm MM, Vallapureddy R, et al. Flow cytometry based monocyte subset analysis accurately distinguishes chronic myelomonocytic leukemia from myeloproliferative neoplasms with associated monocytosis. Blood Cancer J. 2017;7(7):e584.

32. Selimoglu-Buet D, Wagner-Ballon O, Saada V, et al. Characteristic repartition of monocyte subsets as a diagnostic signature of chronic myelomonocytic leukemia. Blood. 2015;125(23):3618-3626.

33. Selimoglu-Buet D, Badaoui B, Benayoun E, et al. Accumulation of classical monocytes defines a subgroup of MDS that frequently evolves into CMML. Blood. 2017;130(6):832-835.

34. Schmidl C, Renner K, Peter K, et al. Transcription and enhancer profiling in human monocyte subsets. Blood. 2014;123(17):e90-99.

35. Talati C, Zhang L, Shaheen G, et al. Monocyte subset analysis accurately distinguishes CMML from MDS and is associated with a favorable MDS prognosis. Blood. 2017;129(13):1881-1883.

36. Wagner-Ballon O, Bettelheim P, Lauf J, et al. ELN iMDS flow working group validation of the monocyte assay for chronic myelomonocytic leukemia diagnosis by flow cytometry. Cytometry B Clin Cytom. 2021 Dec 30. [Epub ahead of print]

37. Hudson CA, Burack WR, Leary PC, Bennett JM. Clinical utility of classical and nonclassical monocyte percentage in the diagnosis of chronic myelomonocytic leukemia. Am J Clin Pathol. 2018;150(4):293-302.

38. Pophali PA, Timm MM, Mangaonkar AA, et al. Practical limitations of monocyte subset repartitioning by multiparametric flow cytometry in chronic myelomonocytic leukemia. Blood Cancer J. 2019;9(9):65.

39. Cargo C, Cullen M, Taylor J, et al. The use of targeted sequencing and flow cytometry to identify patients with a clinically significant monocytosis. Blood. 2019;133(12):1325-1334.

40. Patnaik MM, Barraco D, Lasho TL, et al. DNMT3A mutations are associated with inferior overall and leukemia-free survival in chronic myelomonocytic leukemia. Am J Hematol. 2017;92(1):56-61.

41. Itzykson R, Kosmider O, Renneville A, et al. Clonal architecture of chronic myelomonocytic leukemias. Blood. 2013;121(12):2186-2198.

42. Patnaik MM, Lasho TL, Finke CM, et al. Spliceosome mutations involving SRSF2, SF3B1, and U2AF35 in chronic myelomonocytic leukemia: prevalence, clinical correlates, and prognostic relevance. Am J Hematol. 2013;88(3):201-206.

43. Wudhikarn K, Loghavi S, Mangaonkar AA, et al. SF3B1-mutant CMML defines a predominantly dysplastic CMML subtype with a superior acute leukemia-free survival. Blood Adv. 2020;4(22):5716-5721.

44. Caye A, Strullu M, Guidez F, et al. Juvenile myelomonocytic leukemia displays mutations in components of the RAS pathway and the PRC2 network. Nat Genet. 2015;47(11):1334-1340.

45. DiFilippo EC, Coltro G, Carr RM, et al. Spectrum of abnormalities and clonal transformation in germline RUNX1 familial platelet disorder and a genomic comparative analysis with somatic RUNX1 mutations in MDS/MPN overlap neoplasms. Leukemia. 2020;34(9):2519-2524. 
46. St Martin EC, Ferrer A, Wudhikarn K, et al. Clinical features and survival outcomes in patients with chronic myelomonocytic leukemia arising in the context of germline predisposition syndromes. Am J Hematol. 2021;96(9):E327-E330.

47. Perez Botero J, Oliveira JL, Chen D, et al. ASXL1 mutated chronic myelomonocytic leukemia in a patient with familial thrombocytopenia secondary to germline mutation in ANKRD26. Blood Cancer J. 2015;5(5):e315.

48. Saliba J, Saint-Martin C, Di Stefano A, et al. Germline duplication of ATG2B and GSKIP predisposes to familial myeloid malignancies. Nat Genet. 2015;47(10):1131-1140.

49. Martin ES, Ferrer A, Mangaonkar AA, et al. Spectrum of hematological malignancies, clonal evolution and outcomes in 144 Mayo Clinic patients with germline predisposition syndromes. Am J Hematol. 2021;96(11):1450-1460.

50. Swerdlow SH, Campo E, Harris NL, et al., editors. WHO Classification of Tumors of Haematopoietic and Lymphoid Tissues. Lyon: International Agency for Research on Cancer. 2008.

51. Lucas N, Duchmann M, Rameau P, et al. Biology and prognostic impact of clonal plasmacytoid dendritic cells in chronic myelomonocytic leukemia. Leukemia. 2019;33(10):2466-2480.

52. Swederlow SH, Campo E, Harris NL, et al, editors. WHO Classification of Tumors of the Haematopoietic and Lymphoid Tissue. 4 th ed. Lyon: International Agency for Research on Cancer (IARC), 2008.

53. Bain BJ. What is a promonocyte? Am J Hematol. 2013;88(10):919.

54. Such E, Cervera J, Costa D, et al. Cytogenetic risk stratification in chronic myelomonocytic leukemia. Haematologica. 2011;96(3):375-383.

55. Wassie EA, Itzykson R, Lasho TL, et al. Molecular and prognostic correlates of cytogenetic abnormalities in chronic myelomonocytic leukemia: a Mayo Clinic-French Consortium Study. Am J Hematol. 2014;89(12):1111-1115.

56. Patnaik MM, Rangit V, Lasho TL, et al. A comparison of clinical and molecular characteristics of patients with systemic mastocytosis with chronic myelomonocytic leukemia to $\mathrm{CMML}$ alone. Leukemia. 2018;32(8):1850-1856.

57. Sotlar K, Fridrich C, Mall A, et al. Detection of c-kit point mutation Asp-816 --> Val in microdissected pooled single mast cells and leukemic cells in a patient with systemic mastocytosis and concomitant chronic myelomonocytic leukemia. Leuk Res. 2002;26(11):979-984.

58. DeAngelo DJ, Radia DH, George TI, et al. Safety and efficacy of avapritinib in advanced systemic mastocytosis: the phase 1 EXPLORER trial. Nat Med. 2021;27(12):2183-2191.

59. Patnaik MM, Pophali PA, Lasho TL, et al. Clinical correlates, prognostic impact and survival outcomes in chronic myelomonocytic leukemia patients with the JAK2V617F mutation. Haematologica. 2019;104(6):e236-e239.

60. Patnaik MM, Ketterling RP, Tefferi A. FGFR1 rearranged hematological neoplasms - molecularly defined and clinically heterogeneous. Leuk Lymphoma. 2018;59(7):1520-1522.

61. Padron E, Garcia-Manero G, Patnaik MM, et al. An international data set for CMML validates prognostic scoring systems and demonstrates a need for novel prognostication strategies. Blood Cancer J. 2015;5(7):e333.

62. Patnaik MM, Tefferi A. Chronic myelomonocytic leukemia: focus on clinical practice. Mayo Clin Proc. 2016;91(2):259-272.

63. Onida F, Kantarjian HM, Smith TL, et al. Prognostic factors and scoring systems in chronic myelomonocytic leukemia: a retrospective analysis of 213 patients. Blood. 2002;99(3):840-849.
64. Kantarjian H, O'Brien S, Ravandi F, et al. Proposal for a new risk model in myelodysplastic syndrome that accounts for events not considered in the original International Prognostic Scoring System. Cancer. 2008;113(6):1351-1361.

65. Such E, Germing U, Malcovati L, et al. Development and validation of a prognostic scoring system for patients with chronic myelomonocytic leukemia. Blood. 2013;121(15):3005-3015.

66. Wassie EA, Itzykson R, Lasho TL, et al. Molecular and prognostic correlates of cytogenetic abnormalities in chronic myelomonocytic leukemia: a Mayo Clinic-French Consortium Study. Am J Hematol. 2014;89(12):1111-1115.

67. Patnaik MM, Itzykson R, Lasho TL, et al. ASXL1 and SETBP1 mutations and their prognostic contribution in chronic myelomonocytic leukemia: a two-center study of 466 patients. Leukemia. 2014;28(11):2206-2212.

68. Gagelmann N, Badbaran A, Beelen DW, et al. A prognostic score including mutation profile and clinical features for patients with CMML undergoing stem cell transplantation. Blood Adv. 2021;5(6):1760-1769.

69. Santoriello D, Andal LM, Cox R, D'Agati VD, Markowitz GS. Lysozyme-induced nephropathy. Kidney Int Rep. 2017;2(1):84-88.

70. Fenaux P, Santini V, Spiriti MAA, et al. A phase 3 randomized, placebo-controlled study assessing the efficacy and safety of epoetin-alpha in anemic patients with low-risk MDS. Leukemia. 2018;32(12):2648-2658.

71. Santini V. Anemia as the main manifestation of myelodysplastic syndromes. Semin Hematol. 2015;52(4):348-356.

72. Park S, Greenberg P, Yucel A, et al. Clinical effectiveness and safety of erythropoietin-stimulating agents for the treatment of low- and intermediate-1-risk myelodysplastic syndrome: a systematic literature review. Br J Haematol. 2019;184(2):134-160.

73. Pophali P, Horna P, Lasho TL, et al. Splenectomy in patients with chronic myelomonocytic leukemia: indications, histopathological findings and clinical outcomes in a single institutional series of thirty-nine patients. Am J Hematol. 2018;93(11):1347-1357.

74. Fenaux P, Platzbecker U, Mufti GJ, et al. Luspatercept in patients with lower-risk myelodysplastic syndromes. N Engl J Med. 2020;382(2):140-151.

75. Jabbour E, Short NJ, Montalban-Bravo G, et al. Randomized phase 2 study of low-dose decitabine vs low-dose azacitidine in lower-risk MDS and MDS/MPN. Blood. 2017;130(13):1514-1522.

76. Burgstaller S, Stauder R, Kuehr T, et al. A phase I study of lenalidomide in patients with chronic myelomonocytic leukemia (CMML) - AGMT_CMML-1. Leuk Lymphoma. 2018;59(5):1121-1126.

77. Hoversten K, Vallapureddy R, Lasho T, et al. Nonhepatosplenic extramedullary manifestations of chronic myelomonocytic leukemia: clinical, molecular and prognostic correlates. Leuk Lymphoma. 2018;59(12):2998-3001.

78. Hunter AM, Newman H, Dezern AE, et al. Integrated human and murine clinical study establishes clinical efficacy of ruxolitinib in chronic myelomonocytic leukemia. Clin Cancer Res. 2021;27(22):6095-6105.

79. Peker D, Padron E, Bennett JM, et al. A close association of autoimmune-mediated processes and autoimmune disorders with chronic myelomonocytic leukemia: observation from a single institution. Acta Haematol. 2015;133(2):249-256.

80. Zahid MF, Barraco D, Lasho TL, et al. Spectrum of autoimmune diseases and systemic inflammatory syndromes in patients with chronic myelomonocytic leukemia. Leuk Lymphoma. 2017;58(6):1488-1493.

81. Ramadan $\mathrm{H}$, Duong VH, Al Ali N, et al. Eltrombopag use in 
patients with chronic myelomonocytic leukemia (CMML): a cautionary tale. Clin Lymphoma Myeloma Leuk. 2016;16Suppl:S64-66.

82. Jaiswal S, Ebert BL. Clonal hematopoiesis in human aging and disease. Science. 2019;366(6465):eaan4673.

83. Jaiswal S, Fontanillas P, Flannick J, et al. Age-related clonal hematopoiesis associated with adverse outcomes. N Engl J Med. 2014;371(26):2488-2498.

84. Jaiswal S, Natarajan P, Silver AJ, et al. Clonal hematopoiesis and risk of atherosclerotic cardiovascular disease. N Engl J Med. 2017;377(2):111-121.

85. Niyongere S, Lucas N, Zhou JM, et al. Heterogeneous expression of cytokines accounts for clinical diversity and refines prognostication in CMML. Leukemia. 2019;33(1):205-216.

86. Franzini A, Pomicter AD, Yan D, et al. The transcriptome of CMML monocytes is highly inflammatory and reflects leukemiaspecific and age-related alterations. Blood Adv. 2019;3(20):2949-2961.

87. Padron E, Garcia-Manero G, Patnaik MM, et al. An international data set for CMML validates prognostic scoring systems and demonstrates a need for novel prognostication strategies. Blood Cancer J. 2015;5(7):e333.

88. Pophali P, Matin A, Mangaonkar AA, et al. Prognostic impact and timing considerations for allogeneic hematopoietic stem cell transplantation in chronic myelomonocytic leukemia. Blood Cancer J. 2020;10(11):121.

89. de Witte T, Bowen $D$, Robin M, et al. Allogeneic hematopoietic stem cell transplantation for MDS and CMML: recommendations from an international expert panel. Blood. 2017;129(13):1753-1762.

90. Symeonidis A, van Biezen A, de Wreede L, et al. Achievement of complete remission predicts outcome of allogeneic haematopoietic stem cell transplantation in patients with chronic myelomonocytic leukaemia. A study of the Chronic Malignancies Working Party of the European Group for Blood and Marrow Transplantation. Br J Haematol. 2015;171(2):239-246.

91. Kongtim P, Popat U, Jimenez A, et al. Treatment with hypomethylating agents before allogeneic stem cell transplant improves progression-free survival for patients with chronic myelomonocytic leukemia. Biol Blood Marrow Transplant. 2016;22(1):47-53.

92. Fenaux P, Mufti GJ, Hellstrom-Lindberg E, et al. Efficacy of azacitidine compared with that of conventional care regimens in the treatment of higher-risk myelodysplastic syndromes: a randomised, open-label, phase III study. Lancet Oncol. 2009;10(3):223-232.

93. Silverman LR, Demakos EP, Peterson BL, et al. Randomized controlled trial of azacitidine in patients with the myelodysplastic syndrome: a study of the cancer and leukemia group B. J Clin Oncol. 2002;20(10):2429-2440.

94. Coston T, Pophali P, Vallapureddy R, et al. Suboptimal response rates to hypomethylating agent therapy in chronic myelomonocytic leukemia; a single institutional study of 121 patients. Am J Hematol. 2019;94(7):767-779.

95. Santini V, Allione B, Zini G, et al. A phase II, multicentre trial of decitabine in higher-risk chronic myelomonocytic leukemia. Leukemia. 2018;32(2):413-418.

96. Merlevede J, Droin N, Qin T, et al. Mutation allele burden remains unchanged in chronic myelomonocytic leukaemia responding to hypomethylating agents. Nature Commun. 2016;7:10767.

97. Bejar R, Lord A, Stevenson K, et al. TET2 mutations predict response to hypomethylating agents in myelodysplastic syndrome patients. Blood. 2014;124(17):2705-2712.

98. Coltro G, Mangaonkar AA, Lasho TL, et al. Clinical, molecular, and prognostic correlates of number, type, and functional localization of TET2 mutations in chronic myelomonocytic leukemia (CMML)-a study of 1084 patients. Leukemia. 2019;34(5):1407-1421.

99. Duchmann M, Yalniz FF, Sanna A, et al. Prognostic role of gene mutations in chronic myelomonocytic leukemia patients treated with hypomethylating agents. EBioMedicine. 2018;31:174-181.

100. DiNardo CD, Jonas BA, Pullarkat V, et al. Azacitidine and venetoclax in previously untreated acute myeloid leukemia. N Engl J Med. 2020;383(7):617-629.

101. Sevin M, Debeurme F, Laplane L, et al. Cytokine-like protein 1induced survival of monocytes suggests a combined strategy targeting MCL1 and MAPK in CMML. Blood. 2021;137(24):3390-3402.

102. Saliba AN, Litzow MR, Gangat N, et al. Outcomes of venetoclaxbased therapy in chronic phase and blast transformed chronic myelomonocytic leukemia. Am J Hematol. 2021;96(11):E433-E436.

103. Padron E, Dezern A, Andrade-Campos M, et al. A multiinstitution phase 1 trial of ruxolitinib in patients with chronic myelomonocytic leukemia (CMML). Clin Cancer Res. 2016;22(15):3746-3754.

104. Padron E, Painter JS, Kunigal S, et al. GM-CSF-dependent PSTAT5 sensitivity is a feature with therapeutic potential in chronic myelomonocytic leukemia. Blood. 2013;121(25):5068-5077.

105. Patnaik MM, Sallman DA, Mangaonkar A, et al. Phase 1 study of lenzilumab, a recombinant anti-human GM-CSF antibody, for chronic myelomonocytic leukemia (CMML). Blood. 2020;136(7):909-913.

106. Casolaro A, Golay J, Albanese C, et al. The Polo-like kinase 1 (PLK1) inhibitor NMS-P937 is effective in a new model of disseminated primary CD56+ acute monoblastic leukaemia. PLoS One. 2013;8(3):e58424. 